\title{
Supersymmetric Left-Right models with Gauge Coupling Unification and Fermion Mass Universality
}

\author{
Debasish Borah $^{1, *}$ and Urjit A. Yajnik ${ }^{1,2, \dagger}$ \\ ${ }^{1}$ Department of Physics, Indian Institute of Technology, Bombay, Mumbai - 400076, India \\ ${ }^{2}$ Department of Physics, McGill University, \\ 3600 rue University, Montreal QC H3X 2T8, Canada
}

\begin{abstract}
We explore the unification of gauge couplings and fermion masses in two different types of supersymmetric left-right models, one with Higgs triplets and the other with both Higgs triplets as well as bitriplets. The minimal versions of these models do not give rise to the desired unification and some extra fields have to be added. After such a modification, it is possible in one model to get gauged $B-L$ symmetry to be unbroken down to $\mathrm{TeV}$ scale. We also identify the parameter space at the electroweak scale which gives rise to fermion mass unification at a high scale $M_{G}$. Type I seesaw emerges as the natural explanation of the small neutrino masses in both the models.

PACS numbers: $12.60 . \mathrm{Fr}, 12.60 . \mathrm{Jv}, 14.60 . \mathrm{Pq}$
\end{abstract}

*Electronic address: debasish@phy.iitb.ac.in

${ }^{\dagger}$ Electronic address: yajnik@iitb.ac.in 


\section{INTRODUCTION}

The Left-Right symmetric model [1-5] has always been an appealing extension of the Standard Model of particle physics. In such models parity is spontaneously broken and the smallness of neutrino masses [6-9] arises in a natural way via seesaw mechanism [10-13]. Finally $B-L$ number becomes an abelian gauge charge, which has important simplifying implications for the observed baryon asymmetry of the Universe. Anticipating the embedding of this model in an $S O(10)$ unified theory it is plausible to assume presence of $\mathrm{TeV}$ scale supersymmetry in order to stablize the hierarchy between the electroweak scale and the unification scale. In the class of models to be considered here, generically called Supersymmetric Left-Right (SUSYLR) models, the effective potential of the spontaneously broken theory permits that the $U(1)_{B-L}$ remains unbroken upto low energies, close to TeV scale. This is the possibility we shall assume with a hope of unearthing both supersymmetry and $B-L$ symmetry within collider energy regimes. In this paper we study whether gauge coupling unification remains viable under such conditions, along with a consistent see-saw explanaton for the fermion masses. Within the specific models presented here, we achieve partial success of these goals.

When the non-supersymmetric model of [1-4] is extended to incorporate supersymmetry it is found that the effective potential of the proposed minimal model fails to provide spontaneous parity breaking [14]. One possible direction for ameliaorating this problem is the inclusion of non-renormalizable terms [14][15], or non-perturbative corrections from an additional singlet [16]. In an alternative approach, it was proposed in $[15,17]$ that addition of heavy scalars similar to the $\Delta$ triplets of the non-supersymmetric version, but neutral under $B-L$, avoid the above stated problems and provide spontaneous parity breaking with only renormalizable terms considered. Recently, the spontaneous parity violation was also demonstrated in an alternative supersymmetric Left-Right model [18] where the extra fields added to the minimal field content are a gauge singlet and a bitriplet under $S U(2)_{L} \times S U(2)_{R}$.

These studies remain ad hoc unless additional guiding principles can be used to reduce the variety of models or indeed to single out any one model. In the present work we shall seek input from the requirements of gauge coupling unification and fermion mass universality. However there are additional guiding principles articulated in [19-21]. One concerns the mass scales of the scalars, wherein supersymmetry can give rise to accidentally light 
particles, dubbed "survival of the fittest" phenomenon. The fact that the renormalizable superpotential forbids certain categories of terms in the scalar potential which would have been otherwise permitted by gauge invariance, ensures that certain scalar masses do not receive large corrections from heavier particles in the spectrum. In accordance with this, we shall assume the masses of the scalars to be just as expected from the superpotential. The principle highlighted in the second of the above papers concerns the almost automatic survival of $R$-parity in supersymmetric left-right unification, and as a by-product, the almost pure Type I nature of the see-saw mechanism.

With above discussion in mind we pursue the models of $[15,17]$ and [18] to check the consistency of (i) gauge coupling unification and fermion mass universality, and (ii) the correct order of magnitude for the light neutrino masses, with the exciting possibility of (iii) TeV scale intermediate symmetry breaking. The issue of unification and perturbativity in this class of models has recently been investigated exhaustively in [22]. Our approach is similar in spirit to the study of [23] for a different version of SUSYLR model and with different motivations. Gauge coupling unification issue for the bitriplet Higgs model was studied also in [24]. The present work extends this by the study of evolution of fermion

masses and mixing for this model. A main finding of our paper, that at least for one of the models, gauged $B-L$ charge can remain unbroken down to $3 \mathrm{TeV}$ can have interesting phenomenological consequences.

This paper is organized as follows. In the next section II, we discuss two versions of SUSYLR model [15, 17] as well as [18]. Then in section III we study the gauge coupling unification and in section IV we study the evolution of fermion masses and mixing in both the models. We discuss the neutrino mass phenomenology in section $\mathrm{V}$ and then finally conclude in VI.

\section{TWO POSSIBLE CHOICES FOR THE SUSYLR HIGGS STRUCTURE}

The minimal set of the Higgs fields in the non-supersymmetric Left-Right model consists of a bidoublet $\Phi$ and $S U(2)_{L}$ and $S U(2)_{R}$ triplets $\Delta_{L}$ and $\Delta_{R}$ respectively. In the supersymmetric version, the cancellation of chiral anomalies among the fermionic partners of the triplet Higgs fields $\Delta$ requires introduction of the corresponding triplets $\bar{\Delta}$ with opposite $U(1)_{B-L}$ quantum number. Due to $B-L$ gauge invariance, the $\Delta$ fields do not couple to 
the charged leptons and quarks, but gives majorana masses to neutrinos upon getting a vev ( vacuum expectation value) while the $\bar{\Delta}$ fields do not couple to fermions. The usual fermion masses arise from a bidoublet $\Phi_{u}$. Another bidoublet $\Phi_{d}$ is introduced to avoid the Kobayashi-Maskawa matrix for quarks becoming trivial. The matter supermultiplets of the minimal supersymmetric left-right model are

$$
\begin{gathered}
Q=\left(\begin{array}{l}
u \\
d
\end{array}\right) \sim\left(3,2,1, \frac{1}{3}\right), \quad Q_{c}=\left(\begin{array}{c}
d_{c} \\
u_{c}
\end{array}\right) \sim\left(3^{*}, 1,2,-\frac{1}{3}\right), \\
L=\left(\begin{array}{l}
\nu \\
e
\end{array}\right) \sim(1,2,1,-1), \quad L_{c}=\left(\begin{array}{c}
\nu_{c} \\
e_{c}
\end{array}\right) \sim(1,1,2,1)
\end{gathered}
$$

where the numbers in the brackets denote the quantum numbers under $S U(3)_{c} \otimes S U(2)_{L} \otimes$ $S U(2)_{R} \otimes U(1)_{B-L}$. Also here the convention is such that $L \rightarrow U_{L} L$ under $S U(2)_{L}$, but $L^{c} \rightarrow U_{R}^{*} L^{c}$ under $S U(2)_{R}$. The componentwise content of the scalar components of the Higgs superfields is as follows

$$
\begin{gathered}
\Phi_{1}=\left(\begin{array}{cc}
\phi_{11}^{0} & \phi_{11}^{+} \\
\phi_{12}^{-} & \phi_{12}^{0}
\end{array}\right) \sim(1,2,2,0), \Phi_{2}=\left(\begin{array}{cc}
\phi_{21}^{0} & \phi_{21}^{+} \\
\phi_{22}^{-} & \phi_{22}^{0}
\end{array}\right) \sim(1,2,2,0), \\
\triangle=\left(\begin{array}{cc}
\frac{1}{\sqrt{ } 2} \delta_{L}^{+} & \delta_{L}^{++} \\
\delta_{L}^{0} & -\frac{1}{\sqrt{ } 2} \delta_{L}^{+}
\end{array}\right) \sim(1,3,1,2), \bar{\triangle}=\left(\begin{array}{cc}
\frac{1}{\sqrt{ } 2} \triangle_{L}^{-} & \triangle_{L}^{0} \\
\triangle_{L}^{--} & -\frac{1}{\sqrt{ } 2} \triangle_{L}^{-}
\end{array}\right) \sim(1,3,1,-2), \\
\triangle_{c}=\left(\begin{array}{cc}
\frac{1}{\sqrt{ } 2} \triangle_{R}^{-} & \triangle_{R}^{--} \\
\triangle_{R}^{0} & -\frac{1}{\sqrt{ } 2} \triangle_{R}^{-}
\end{array}\right) \sim(1,1,3,-2), \bar{\triangle}_{c}=\left(\begin{array}{cc}
\frac{1}{\sqrt{ } 2} \delta_{R}^{+} & \delta_{R}^{0} \\
\delta_{R}^{++} & -\frac{1}{\sqrt{ } 2} \delta_{R}^{+}
\end{array}\right) \sim(1,1,3,2)
\end{gathered}
$$

Under left-right symmetry the fields transform as

$$
Q \leftrightarrow Q_{c}^{*}, \quad L \leftrightarrow L_{c}^{*}, \quad \Phi \leftrightarrow \Phi^{\dagger}, \quad \triangle \leftrightarrow \triangle_{c}^{*}, \quad \bar{\triangle} \leftrightarrow \bar{\triangle}^{*}{ }_{c}
$$

It turns out that left-right symmetry imposes rather strong constraints on the ground state of this model. It was pointed out by Kuchimanchi and Mohapatra [14] that there is no spontaneous parity breaking for this minimal choice of Higgs in the supersymmetric leftright model and as such the ground state remains parity symmetric. If parity odd singlets are introduced to break this symmetry [25], then it was shown [14] that the charge-breaking vacua have a lower potential than the charge-preserving vacua and as such the ground state does not conserve electric charge. Breaking $R$ parity was another possible solution [14] 
to this dilemma of breaking parity symmetry. For instance, it was shown recently in [26] that both Left-Right symmetry and R-parity can be broken simultaneously by right handed sneutrino vev.

A solution to this impasse without breaking $R$ parity is to add two new triplet superfields $\Omega(1,3,1,0), \Omega_{c}(1,1,3,0)$ where under parity symmetry $\Omega \leftrightarrow \Omega_{c}^{*}$. This possibility has been explored extensively in $[15,17,27,28]$, which we refer to as the Aulakh-Bajc-Melfo-RasinSenjanovic (ABMRS) model. Another possibility is to add a Higgs bitriplet $\eta(1,3,3,0)$ and a parity odd singlet $\rho(1,1,1,0)[18]$ which also breaks parity spontaneously keeping R-parity conserved. We call this simply the bitriplet model. We discuss both these models below.

\section{A. The ABMRS model}

As shown in the paper [17], the superpotential for this model is given by

$$
\begin{aligned}
W & =h_{l}^{(i)} L^{T} \tau_{2} \Phi_{i} \tau_{2} L_{c}+h_{q}^{(i)} Q^{T} \tau_{2} \Phi_{i} \tau_{2} Q_{c}+i f L^{T} \tau_{2} \triangle L+i f^{*} L_{c}^{T} \tau_{2} \triangle_{c} L_{c} \\
& +m_{\triangle} \operatorname{Tr} \triangle \bar{\triangle}+m_{\Delta}^{*} \operatorname{Tr} \triangle_{c} \bar{\triangle}_{c}+\frac{m_{\Omega}}{2} \operatorname{Tr} \Omega^{2}+\frac{m_{\Omega}^{*}}{2} \operatorname{Tr} \Omega_{c}^{2} \\
& +\mu_{i j} \operatorname{Tr} \tau_{2} \Phi_{i}^{T} \tau_{2} \Phi_{j}+a \operatorname{Tr} \triangle \Omega \bar{\triangle}+a^{*} \operatorname{Tr} \triangle_{c} \Omega_{c} \bar{\triangle}_{c} \\
& +\alpha_{i j} \operatorname{Tr} \Omega \Phi_{i} \tau_{2} \Phi_{j}^{T} \tau_{2}+\alpha_{i j}^{*} \operatorname{Tr} \Omega_{c} \Phi_{i}^{T} \tau_{2} \Phi_{j} \tau_{2}
\end{aligned}
$$

where $h_{q, l}^{(i)}=h_{q, l}^{(i) \dagger}, \mu_{i j}=\mu_{j i}=\mu_{i j}^{*}, \alpha_{i j}=-\alpha_{j i}$ and $f, h$ are symmetric matrices. It is clear from the above superpotential that the theory has no baryon or lepton number violating terms. The Higgs fields either have $B-L=2$ or 0 . As such the spontaneous symmetry breaking automatically preserves $R$-parity defined by $(-1)^{3(B-L)+2 S}$. Denoting the vev's of the neutral components of Higgs fields $\Omega_{c}$ and $\triangle_{c}$ to be $\omega_{R}$ and $v_{R}$, the supersymmetric vacua obtained from the F-flatness conditions give the relationships

$$
\omega_{R}=-\frac{m_{\triangle}}{a} \equiv-M_{R}, \quad v_{R}=\sqrt{\frac{2 m_{\triangle} m_{\Omega}}{a^{2}}} \equiv M_{B-L}
$$

The structure of $\Omega$ vev gives $S U(2)_{R} \rightarrow U(1)_{R}$. Thus if $v_{R}<\omega_{R}$ then the electroweak $U(1)_{Y}$ results only after the $\Delta$ fields get vev. The resulting symmetry breaking sequence in this case is

$$
S U(2)_{L} \otimes S U(2)_{R} \otimes U(1)_{B-L} \underline{\left\langle\Omega_{c}\right\rangle} S U(2)_{L} \otimes U(1)_{R} \otimes U(1)_{B-L}\left\langle\triangle_{c}\right\rangle S U(2)_{L} \otimes U(1)_{Y}
$$


The choice $v_{R}<\omega_{R}$ also provides unambiguous parity breaking from an early stage. The two scales in question can be kept distinct, by ensuring $M_{B-L} \ll M_{R}$ which can be achieved by choosing $m_{\triangle} \gg m_{\Omega}$. A possibility for avoiding proliferation of new mass scales is to assume $m_{\Omega}=0$ in the superpotential, by proposing an R-symmetry [17]. Then the physical value of $m_{\Omega}$ in the above relations can be assumed to arise from soft supersymmetry breaking terms, and hence of $M_{E W}$ scale. This then implies the relation $M_{B-L}^{2}=M_{E W} M_{R}$ which relates different symmetry breaking scales.

In [17], the vacumm structure was analysed from $F$ flatness conditions. For our purpose we consider the full scalar potential for the model given by

$$
V=|F|^{2}+D^{a} D^{a} / 2+V_{\text {soft }}
$$

where $F=\frac{\partial W}{\partial \phi}, D^{a}=-g\left(\phi_{i}^{*} T_{i j}^{a} \phi_{j}\right)$, g is gauge coupling constants, $T^{a}$ is the generators of the corresponding gauge group and $\phi$ 's are chiral superfields, and $V_{\text {soft }}$ denotes all the soft supersymmetry breaking terms. We denote $\langle\triangle\rangle=v_{L},\left\langle\triangle_{c}\right\rangle=v_{R},\langle\Omega\rangle=\omega_{L},\left\langle\Omega_{c}\right\rangle=\omega_{R}$, $\langle\Phi\rangle=v$. The soft terms can be ignored for pursuing the high scale physics, and the minimization of the scalar potential terms $V_{F}+V_{D}$ with respect to $v_{L}, v_{R}, \omega_{L}, \omega_{R}$ and $v$ respectively gives

$$
\begin{gathered}
v_{L}\left(m_{\triangle}^{2}+a \omega_{L} m_{\triangle}+a^{2} \omega_{L}^{2}+m_{\Omega} \omega_{L}+a v_{L}^{2}+\alpha v^{2}+g^{2} v_{L}^{2}\right)=0 \\
v_{R}\left(m_{\triangle}^{2}-a \omega_{R} m_{\triangle}+a^{2} \omega_{R}^{2}-m_{\Omega} \omega_{R}+a v_{R}^{2}+\alpha v^{2}+g^{2} v_{R}^{2}\right)=0 \\
a^{2} \omega_{L} v_{L}^{2}+a m_{\triangle} v_{L}^{2}+\omega_{L} m_{\Omega}^{2}+m_{\Omega} a v_{L}^{2}+\alpha v^{2} m_{\Omega}+v^{2} \alpha^{2} \omega_{R}+g^{2} \omega_{L}^{3}=0 \\
a^{2} \omega_{R} v_{R}^{2}-a m_{\triangle} v_{R}^{2}+\omega_{R} m_{\Omega}^{2}-m_{\Omega} a v_{R}^{2}+\alpha v^{2} m_{\Omega}+v^{2} \alpha^{2} \omega_{R}+g^{2} \omega_{R}^{3}=0 \\
v \alpha\left(m_{\Omega} \omega_{L}+a v_{L}^{2}+\omega_{R} m_{\Omega}-a v_{R}^{2}+\mu\left(\omega_{L}+\omega_{R}\right)+\left(\omega_{L}+\omega_{R}\right)^{2} \alpha\right)+g^{2} v^{3}=0
\end{gathered}
$$

Note that it is sufficient for phenomenology to choose $v_{R} \neq 0$ and then it is natural to set $v_{L}=0$. In the non-supersymmetric version, $v_{L}$ gets mixed with $v_{R}$ at tree level, and its value though small is not negligible for the purpose of neutrino masses. This possibility is precluded here by the restriction imposed by supersymmetry. In turn this ensures pure Type I see-saw for the neutrino mass, assuming loop corrections to $v_{L}$ remain small. This also goes well with the requirements $v_{L}, \omega_{L} \ll M_{E W}$ so as not to affect the Standard Model $\rho$ parameter. 
Our purpose here is to study the possibility of a $\mathrm{TeV}$ scale $U(1)_{B-L}$ breaking scale and a high $S U(2)_{R}$ breaking scale which can give rise to gauge coupling unification as well as small neutrino mass from seesaw. Thus we choose $v_{R} \sim \mathrm{TeV}, \omega_{R} \sim M_{G}$, where $M_{G}$ is the scale where the couplings unify. This can be ensured by taking $m_{\Omega} \sim \mathrm{TeV}$ and $m_{\triangle} \sim M_{G}$, with $M_{G}$ is expected to be $M_{R}$ introduced in Eq. (8). With this choice, in the next section we study how the gauge couplings as well as fermion masses and mixings evolve under the renormalization group equations (RGE).

\section{B. The Bitriplet Model}

The model above utilised two mutually unrelated superfields $\Omega$ and $\Omega_{c}$. We may attempt to achieve the same effect by invoking a bitriplet superfield $\eta(1,3,3,0)$. This while separating the $M_{R}$ and the $M_{B-L}$ scales as before, does not however succeed in providing spontaneous parity breaking. We are then led to add a parity odd singlet $\rho(1,1,1,0)$ to the particle content of minimal SUSYLR model [18]. The superpotential with this Higgs content is

$$
\begin{aligned}
& W=f \eta_{\alpha i} \triangle_{\alpha} \triangle_{i}^{c}+f^{*} \eta_{\alpha i} \bar{\triangle}_{\alpha} \bar{\triangle}_{i}^{c}+\lambda_{1} \eta_{\alpha i} \Phi_{a m} \Phi_{b n}\left(\tau^{\alpha} \epsilon\right)_{a b}\left(\tau^{i} \epsilon\right)_{m n}+m_{\eta} \eta_{\alpha i} \eta_{\alpha i} \\
& \quad+M_{\triangle}\left(\triangle_{\alpha} \bar{\triangle}_{\alpha}+\triangle_{i}^{c} \bar{\triangle}_{i}^{c}\right)+\mu \epsilon_{a b} \Phi_{b m} \epsilon_{m n} \Phi_{a n}+m_{\rho} \rho^{2}+\lambda_{2} \rho\left(\triangle_{\alpha} \bar{\triangle}_{\alpha}-\triangle_{i}^{c} \bar{\triangle}_{i}^{c}\right)
\end{aligned}
$$

where $\alpha, a, b$ are $S U(2)_{L}$ and $i, m, n$ are $S U(2)_{R}$ indices. The symmetry breaking pattern in this model is

$$
\begin{gathered}
S U(2)_{L} \times S U(2)_{R} \times U(1)_{B-L} \times P \quad \stackrel{\langle\eta\rangle}{\longrightarrow} S U(2)_{L} \times S U(2)_{R} \times U(1)_{B-L} \\
\underline{\left\langle\triangle_{c}\right\rangle} S U(2)_{L} \times U(1)_{Y} \stackrel{\langle\Phi\rangle}{\longrightarrow} U(1)_{e m}
\end{gathered}
$$

Denoting the vev's as $\left\langle\triangle_{-}\right\rangle=\left\langle\bar{\triangle}_{+}\right\rangle=v_{L},\left\langle\triangle_{+}^{c}\right\rangle=\left\langle\bar{\triangle}_{-}^{c}\right\rangle=v_{R},\left\langle\Phi_{+-}\right\rangle=v,\left\langle\Phi_{-+}\right\rangle=$ $v^{\prime},\left\langle\eta_{+-}\right\rangle=u_{1},\left\langle\eta_{-+}\right\rangle=u_{2},\left\langle\eta_{00}\right\rangle=u_{0}$. The scalar potential is $V=V_{F}+V_{D}+V_{\text {soft }}$ where $V_{F}=\left|F_{i}\right|^{2}, F_{i}=-\frac{\partial W}{\partial \phi}$ is the F-term scalar potential, $V_{D}=D^{a} D^{a} / 2, D^{a}=-g\left(\phi_{i}^{*} T_{i j}^{a} \phi_{j}\right)$ is the D-term of the scalar potential and $V_{\text {soft }}$ is the soft supersymmetry breaking terms in the scalar potential. Ignoring the soft terms as before for analysis of the high scale physics we have

$$
\begin{aligned}
& \frac{\partial V}{\partial v_{L}}=\mu_{L}^{2}\left(2 v_{L}\right)+2 \lambda_{2}^{2} v_{L}\left(v_{L}^{2}-v_{R}^{2}\right)+2\left(f u_{1}+f^{*} u_{2}\right) M_{\triangle} v_{R} \\
& \quad+v_{R}\left(f+f^{*}\right)\left[2 m_{\eta}\left(u_{1}+u_{2}+u_{3}\right)+\lambda_{1} v^{2}+v_{L} v_{R}\left(f+f^{*}\right)\right]=0
\end{aligned}
$$




$$
\begin{aligned}
& \frac{\partial V}{\partial v_{R}}=\mu_{R}^{2}\left(2 v_{R}\right)-2 \lambda_{2}^{2} v_{R}\left(v_{L}^{2}-v_{R}^{2}\right)+2\left(f u_{1}+f^{*} u_{2}\right) M_{\triangle} v_{L} \\
& \quad+v_{L}\left(f+f^{*}\right)\left[2 m_{\eta}\left(u_{1}+u_{2}+u_{3}\right)+\lambda_{1} v^{2}+v_{L} v_{R}\left(f+f^{*}\right)\right]=0
\end{aligned}
$$

Where the effective mass terms $\mu_{L}^{2}, \mu_{R}^{2}$ are given by

$$
\begin{aligned}
& \mu_{L}^{2}=\left(M_{\triangle}+\lambda_{2} s\right)^{2}+\lambda_{2} m_{\rho} s+\frac{1}{2}\left(f^{2} u_{1}^{2}+f^{* 2} u_{2}^{2}\right) \\
& \mu_{R}^{2}=\left(M_{\triangle}-\lambda_{2} s\right)^{2}-\lambda_{2} m_{\rho} s+\frac{1}{2}\left(f^{2} u_{1}^{2}+f^{* 2} u_{2}^{2}\right)
\end{aligned}
$$

Thus after the singlet field $\rho$ acquires a vev the degeneracy of the Higgs triplets goes away and the left handed triplets being very heavy get decoupled whereas the right handed triplets can be as light as $1 \mathrm{TeV}$ by appropriate fine tuning in the above two expressions. Assuming $v_{L} \ll v_{R} \ll m_{\rho}, m_{s}$ we get from equations (16), (17):

$$
v_{L}=\frac{-v_{R}\left[M_{\triangle} u_{2} f^{*}+m_{\eta}\left(u_{2}+u_{3}\right)\left(f+f^{*}\right)+u_{1}\left(f M_{\triangle}+m_{\eta}\left(f+f^{*}\right)\right]\right.}{2 m_{\rho} s \lambda_{2}+4 M_{\triangle} s \lambda_{2}}
$$

To understand this relation let us assume $s \sim M_{\triangle} \sim m_{\eta} \sim m_{\rho}$ collectively denoted by $M_{R}$ to be large, and $u_{1} \sim u_{2} \sim u_{3}$ denoted $u$ to be small. The above relation then reads, ignoring dimensionless numbers,

$$
v_{L} \approx v_{R} \times \frac{u}{M_{R}}
$$

We must take the vev of the bitriplet $u \ll M_{Z}$ so as not to affect the Standard Model $\rho$ parameter. On the other hand $v_{L}$ which enters the see-saw formula has maximally allowed value $\sim \mathrm{eV}$. Thus if we want $v_{R}$ to be low, $\sim 1 \mathrm{TeV}$, possibly giving rise to collider signatures, then the above relation when saturated requires the scale of parity breaking to be kept low compared to GUT scale, $M_{R} \sim 10^{10} \mathrm{GeV}$. But we shall see that gauge coupling unification forces $M_{R}$ to be much higher. This leaves a large parameter space for the possible values of $v_{L}$ and $v_{R}$, such that they remain phenomenologically accessible. In particular, retaining Type I see-saw for neutrino masses remains natural.

\section{GAUGE COUPLING UNIFICATION}

The one-loop renormalization group evolution equations [29] are given by

$$
\mu \frac{d g_{i}}{d \mu}=\beta_{i}\left(g_{i}\right)=\frac{g_{i}^{3}}{16 \pi^{2}} b_{i}
$$


Defining $\alpha_{i}=g_{i}^{2} /(4 \pi)$ and $t=\ln \left(\mu / \mu_{0}\right)$ and the most general renormalization group equation above becomes

$$
\frac{d \alpha_{i}^{-1}}{d t}=-\frac{b_{i}}{2 \pi}
$$

The one-loop beta function is given by

$$
\beta_{i}\left(g_{i}\right)=\frac{g_{i}^{3}}{16 \pi^{2}}\left[-\frac{11}{3} \operatorname{Tr}\left[T_{a}^{2}\right]+\frac{2}{3} \sum_{f} \operatorname{Tr}\left[T_{f}^{2}\right]+\frac{1}{3} \sum_{s} \operatorname{Tr}\left[T_{s}^{2}\right]\right]
$$

where $f$ means the fermions and $s$ means the scalars. For $S U(N), \operatorname{Tr}\left[T_{a}^{2}\right]=N$ and $\operatorname{Tr}\left[T_{i} T_{i}\right]=$ $\frac{1}{2}$. For a supersymmetric model the most general beta function is given by

$$
\beta_{i}\left(g_{i}\right)=\frac{g_{i}^{3}}{16 \pi^{2}}\left[-3 \operatorname{Tr}\left[T_{a}^{2}\right]+\sum_{f} \operatorname{Tr}\left[T_{f}^{2}\right]+\sum_{s} \operatorname{Tr}\left[T_{s}^{2}\right]\right]
$$

The one-loop renormalization group evolutions(RGE) for the masses in SUSYLR model have already been calculated analytically in [30] whereas the same for Minimal Supersymmetric Standard Model (MSSM) can be found in [31]. A very recent analysis on the evolution of fermion masses and mixing was carried out in [23]. We will use the analytical results from these references to study the gauge couplings, fermion mass and mixing evolution in both ABMRS and the bitriplet model below.

\section{A. The ABMRS model}

For the particle content of the ABMRS model we calculate the beta functions as follows

- Below the SUSY breaking scale $M_{\text {susy }}$ the beta functions are same as those of the standard model

$$
b_{s}=-11+\frac{4}{3} n_{g}, \quad b_{2 L}=-\frac{22}{3}+\frac{4}{3} n_{g}+\frac{1}{6} n_{b}, \quad b_{Y}=\frac{4}{3} n_{g}+\frac{n_{b}}{10}
$$

- For $M_{\text {susy }}<M<M_{B-L}$, the beta functions are same as those of the MSSM

$$
b_{s}=-9+2 n_{g}, \quad b_{2 L}=-6+2 n_{g}+\frac{n_{b}}{2}, \quad b_{Y}=2 n_{g}+\frac{3}{10} n_{b}
$$

- For $M_{B-L}<M<M_{R}$ the beta funtions are

$$
\begin{gathered}
b_{s}=-9+2 n_{g}, \quad b_{2 L}=-6+2 n_{g}+\frac{n_{b}}{2}+2 n_{\Omega} \\
b_{1 R}=2 n_{g}+\frac{n_{b}}{2}+2 n_{\Omega}, \quad b_{B-L}=2 n_{g}
\end{gathered}
$$


- For $M_{R}<M<M_{G U T}$ the beta functions are

$$
\begin{gathered}
b_{s}=-9+2 n_{g}, \quad b_{2 L}=-6+2 n_{g}+n_{b}+2 n_{\Omega}+2 n_{\triangle} \\
b_{2 R}=-6+2 n_{g}+n_{b}+2 n_{\Omega}+2 n_{\triangle}, \quad b_{B-L}=2 n_{g}+9 n_{\triangle}
\end{gathered}
$$

Where $n_{g}=3, n_{b}=2, n_{\Omega}=1, n_{\triangle}=2$ are the number of generations, number of bidoublets, number of $\Omega$ and number of $\triangle$ respectively.

It is found that with just the particle content of the SUSYLR model discussed above, the gauge couplings do not unify because of too fast a running of the coupling $\alpha_{3 c}$. Additional colored superfields are needed to achieve unification. We find that two pairs of extra superfields $\chi_{1,2}\left(3,1,1,-\frac{2}{3}\right), \bar{\chi}_{1,2}\left(\overline{3}, 1,1, \frac{2}{3}\right)$, singlet under the $S U(2)_{L, R}$ are needed for the gauge couplings to unify. Each of these extra superfields contributes $\frac{1}{2}$ to both the beta functions $b_{s}$ and $b_{B-L}$ and does not affect the other beta functions, while the vectorlike choice of charges ensures no anomalies. Contributions of a variety of such new superfields to the beta functions were calculated in [22] and our result is in agreement with them. Interestingly, while this many additional fields are just sufficient to achieve the required goal, any additional added fields will drive the coupling into the Landau pole. The resulting unification is shown in figure 1. These extra superfields can naturally be accommodated within SO(10) GUT representations, either $\mathbf{1 2 0}$ or $\overline{\mathbf{1 2 6}}$. Here we assume that masses of these extra superfields can be as low as the $U(1)_{B-L}$ breaking scale.

\section{B. The Bitriplet Model}

It is found that the gauge couplings do not unify for the minimal field content of the bitriplet model. Similar to the AMBRS model, it is adequate to add four heavy colored

superfields $\chi_{1,2}\left(3,1,1,-\frac{2}{3}\right), \bar{\chi}_{1,2}\left(\overline{3}, 1,1, \frac{2}{3}\right)$. And these are then required to decouple below the $S U(2)_{R}$ breaking scale $M_{R}$. The beta functions above $M_{R}$ are

- For $M_{R}<M<M_{\rho}$ the beta functions are

$$
\begin{gathered}
b_{s}=-9+2 n_{g}+\frac{n_{\chi}}{2}, \quad b_{2 L}=-6+2 n_{g}+n_{b}+2 n_{\eta} \\
b_{2 R}=-6+2 n_{g}+n_{b}+2 n_{\triangle}+2 n_{\eta}, \quad b_{B-L}=2 n_{g}+\frac{9}{2} n_{\triangle}+\frac{n_{\chi}}{2}
\end{gathered}
$$




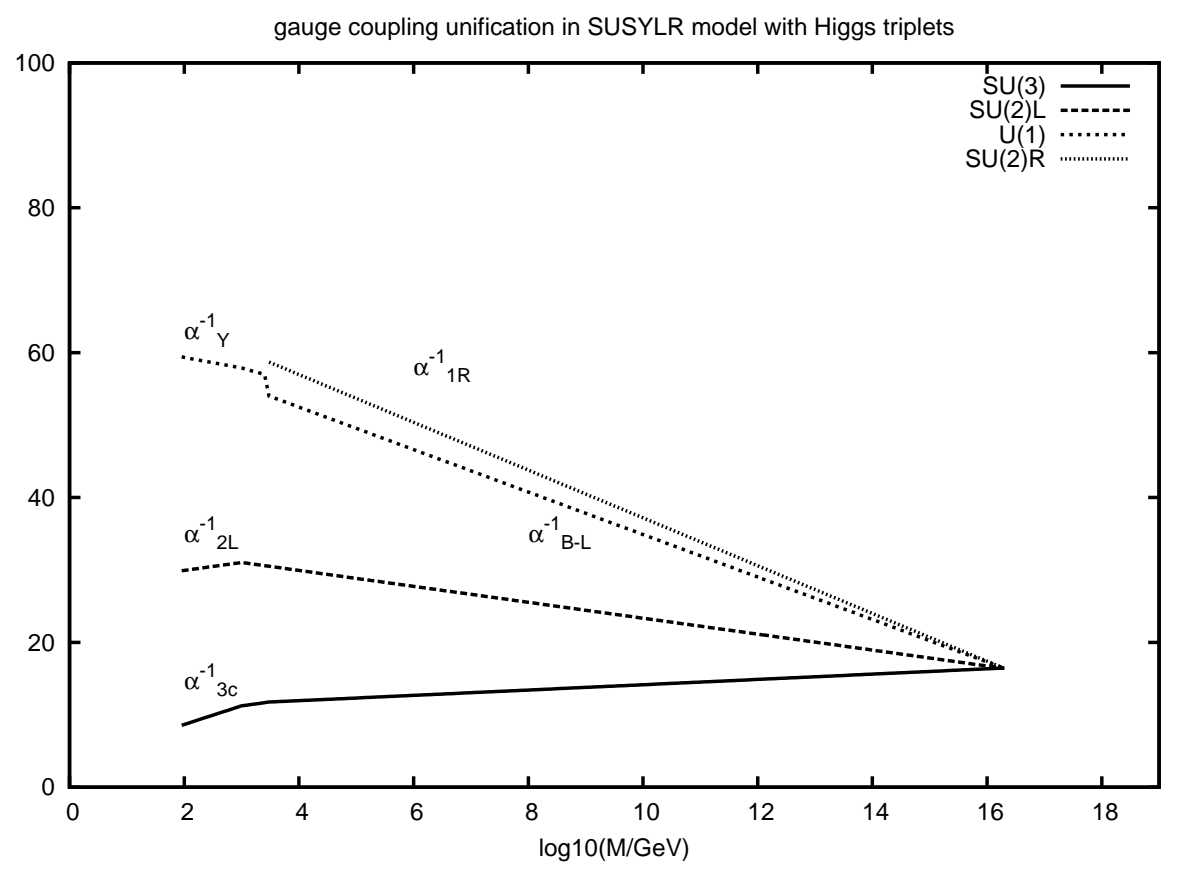

FIG. 1: Gauge coupling unification in the ABMRS model with two extra pairs of colored superfields $\chi_{1,2}\left(3,1,1,-\frac{2}{3}\right), \bar{\chi}_{1,2}\left(\overline{3}, 1,1, \frac{2}{3}\right), M_{S U S Y}=1 \mathrm{TeV}, m_{\Omega}=M_{B-L}=3 \mathrm{TeV}, m_{\triangle}=M_{R}=M_{\mathrm{GUT}}=$ $2 \times 10^{16} \mathrm{GeV}$. The extra superfields decouple below $M_{B-L}$.

- For $\langle\rho\rangle<M<M_{G U T}$ the beta functions are

$$
\begin{gathered}
b_{s}=-9+2 n_{g}+\frac{n_{\chi}}{2}, \quad b_{2 L}=-6+2 n_{g}+n_{b}+2 n_{\triangle}+2 n_{\eta} \\
b_{2 R}=-6+2 n_{g}+n_{b}+2 n_{\triangle}+2 n_{\eta}, \quad b_{B-L}=2 n_{g}+9 n_{\triangle}+\frac{n_{\chi}}{2}
\end{gathered}
$$

where $n_{\triangle}=2, n_{\chi}=4, n_{g}=3, n_{b}=2, n_{\eta}=1$. Using the same initial values and normalization relations as before we arrive at the gauge coupling unification, an essential result of [24], as shown in Fig. 2. Here the unification scale is the same as the D-parity breaking scale. Similar to the case with just Higgs triplets, here also lower value of $M_{R}$ makes the unification look worse. Thus although minimization of the scalar potential allows the possibility of a TeV scale $M_{R}$ in this model, the requirement of gauge coupling unification rules out such a possibility. 


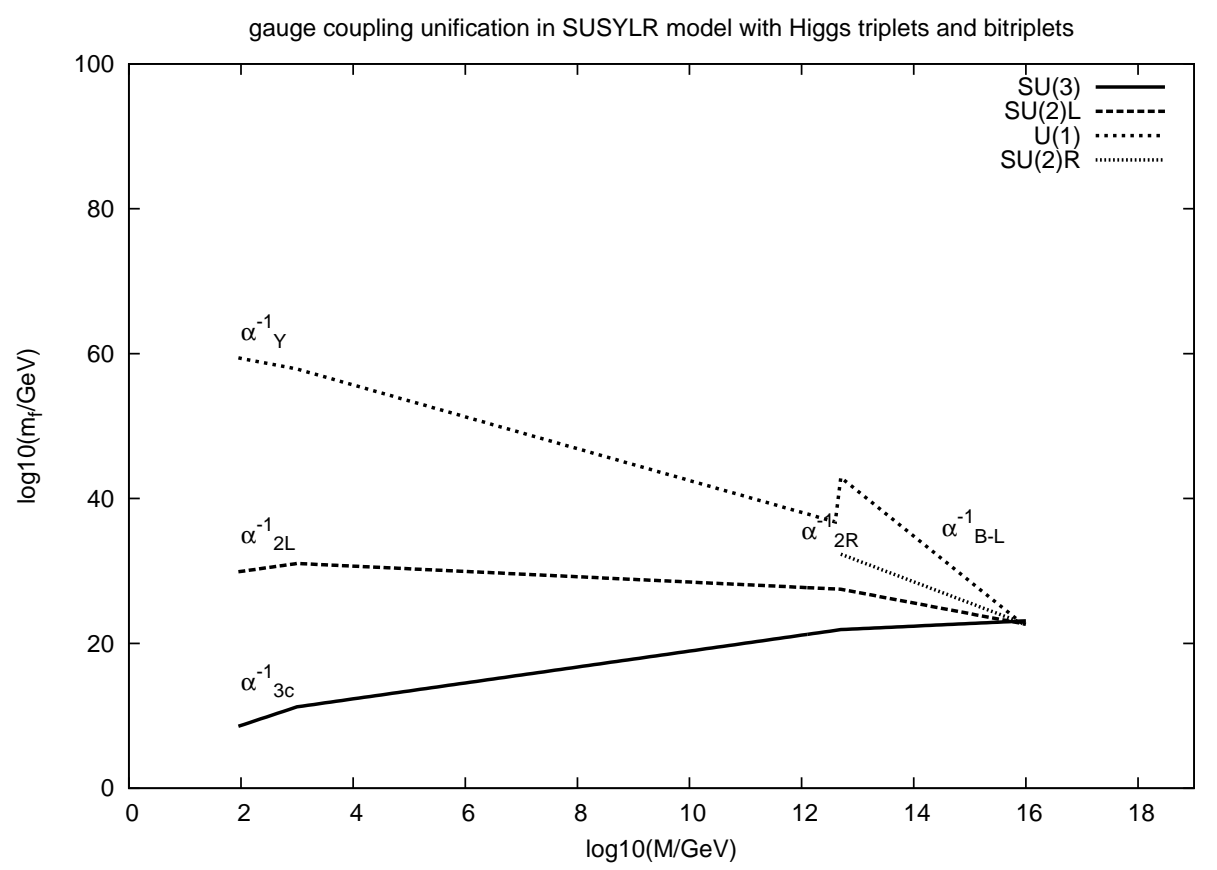

FIG. 2: Gauge coupling unification in the bitriplet model with two extra pairs of colored superfields $\chi_{1,2}\left(3,1,1,-\frac{2}{3}\right), \bar{\chi}_{1,2}\left(\overline{3}, 1,1, \frac{2}{3}\right), M_{\text {susy }}=1 \mathrm{TeV}, M_{R}=5 \times 10^{12} \mathrm{GeV}, M_{\mathrm{GUT}}=M_{\rho}=10^{16} \mathrm{GeV}$. The extra superfields decouple below the scale $M_{R}$.

\section{RUNNING FERMION MASSES AND MIXINGS}

Assuming that the gauge coupling unification is achieved due to the presence of the additional colored multiplets as discussed in the previous section, we consider the question of fermion mass universality and fermion mixing. We also do this in the same context as in the previous section by fixing the intermediate symmetry breaking scales to those which gave rise to gauge coupling unification as in Fig. 1 and Fig. 2. To analyse the fermion mass running we consider all the leptonic yukawa couplings to be diagonal for simplicity. We take the initial values of the masses and mixing parameters at the electroweak scale from [32]. After fixing all these, we are still left with the freedom of choosing the couplings $f, f^{*}$ at the electroweak scale and the ratio of the two electroweak vevs $\left\langle\Phi_{1}\right\rangle=\operatorname{diag}\left(v_{1}, 0\right),\left\langle\Phi_{2}\right\rangle=\operatorname{diag}\left(0, v_{2}\right)$ which we denote as $\tan \beta=\frac{v_{1}}{v_{2}}$. Within the context of a simple analysis, we assume $f, f^{*}$ to be diagonal, and proportional to the identity matrix at the electroweak scale.

Before presenting the general answer for the allowed range of parameters $|f|$ and $\tan \beta$ consistent with $b-\tau$ unification, let us consider specific successful pairs of values which 
work for each of the models. The predictions for fermion masses and mixings at the GUT scale for ABMRS model, with $|f|=0.55$ and $\tan \beta=10$, and for the bitriplet model with $|f|=0.90$ and $\tan \beta=10$ are shown in table I. The running of fermion masses in detail are shown in Fig. 3 for the ABMRS model and in Fig. 4 for the bitriplet model.

TABLE I: Running Fermion masses in SUSYLR model for $\tan \beta=10$

\begin{tabular}{|c|c|c|c|}
\hline Fermion Masses & $\begin{array}{c}M=M_{Z} \\
\text { PDG [32] }\end{array}$ & $\begin{array}{c}M=M_{G}(\mathrm{ABMRS}) \\
(|f|=0.55)\end{array}$ & $\begin{array}{c}M=M_{G} \text { (Bitriplet) } \\
(|f|=0.90)\end{array}$ \\
\hline$m_{u}(\mathrm{MeV})$ & $2.33_{-0.45}^{+0.42}$ & 2.629 & 1.635 \\
$m_{d}(\mathrm{MeV})$ & $4.69_{-0.66}^{+0.60}$ & 2.659 & 2.905 \\
$m_{c}(\mathrm{MeV})$ & $677_{-61}^{+56}$ & 383.654 & 403.89 \\
$m_{s}(\mathrm{MeV})$ & $93.4_{-13.0}^{+11.8}$ & 52.924 & 57.881 \\
$m_{t}(\mathrm{GeV})$ & $181 \pm 13$ & 124.511 & 128.69 \\
$m_{b}(\mathrm{GeV})$ & $3.0 \pm 0.11$ & 2.542 & 2.138 \\
$m_{e}(\mathrm{MeV})$ & $0.48684727 \pm 0.14 \times 10^{-6}$ & 0.5953 & 0.5549 \\
$m_{\mu}(\mathrm{MeV})$ & $102.75138 \pm 3.3 \times 10^{-4}$ & 124.22 & 116.823 \\
$m_{\tau}(\mathrm{GeV})$ & $1.74669_{-0.00027}^{+0.00030}$ & 2.615 & 2.046 \\
\hline
\end{tabular}

At the GUT scale the ratios of fermion masses come out to be

$$
\frac{m_{b}}{m_{\tau}}=0.9720, \quad \frac{3 m_{s}}{m_{\mu}}=1.2781, \quad \frac{m_{d}}{3 m_{e}}=1.4888
$$

for the ABMRS model, and

$$
\frac{m_{b}}{m_{\tau}}=1.0449, \quad \frac{3 m_{s}}{m_{\mu}}=1.4863, \quad \frac{m_{d}}{3 m_{e}}=1.7450
$$

for the bitriplet model. These ratios are expected to be unity in a grand unified theory [33]. The mismatch in the values, especially that for the lighter quarks and leptons is expected to be corrected by the incorporation of various threshold effects [34-40]. We also study the running of Cabbibo Kobayashi Maskawa (CKM) elements and their predicted values at the 
TABLE II: Running CKM elements in SUSYLR model for $\tan \beta=10$

\begin{tabular}{|c|c|c|c|}
\hline CKM elements & $\begin{array}{c}M=M_{Z} \\
\text { PDG [32] }\end{array}$ & $\begin{array}{c}M=M_{G} \text { (ABMRS) } \\
(|f|=0.55)\end{array}$ & $\begin{array}{c}M=M_{G} \text { (Bitriplet) } \\
(|f|=0.90)\end{array}$ \\
\hline$V_{u d}$ & 0.9742 & 0.9780 & 0.9777 \\
$V_{u s}$ & 0.2256 & $0.208-0.0008 i$ & $0.210-0.0008 i$ \\
$V_{u b}$ & $0.0013-0.0033 i$ & $0.0006-0.0021 i$ & $0.0006-0.0021 i$ \\
$V_{c d}$ & $-0.2255-0.0001 i$ & $-0.208-0.0009 i$ & $-0.210-0.0008 i$ \\
$V_{c s}$ & 0.9734 & 0.9777 & 0.9773 \\
$V_{c b}$ & 0.0415 & 0.0269 & 0.0268 \\
$V_{t d}$ & $0.0081-0.0032 i$ & $0.0072-0.0029 i$ & $0.0072-0.0029 i$ \\
$V_{t s}$ & $-0.0407-0.0007 i$ & $-0.0378-0.0006 i$ & $-0.0378-0.0006 i$ \\
$V_{t b}$ & 0.9991 & 0.9851 & 0.9850 \\
\hline
\end{tabular}

GUT scale are mentioned in table II.

These results can be generalised by allowing a variation of $m_{b} / m_{\tau}\left(M_{G}\right)$ with respect to the initial choices of $\tan \beta$ and the yukawa coupling $|f|$. We plot the allowed range of parameters $(|f|, \tan \beta)$ for which $\left|m_{b} / m_{\tau}\left(M_{G}\right)-1\right|<0.1,\left|\frac{3 m_{s}}{m_{\mu}}\left(M_{G}\right)-1\right|<0.5,\left|\frac{m_{d}}{3 m_{e}}\left(M_{G}\right)-1\right|<0.5$. Usually there is far less discrepancy in the case of third generation fermion universality at the unification scale, and hence we allow variation of $10 \%$ error in its value, expecting the discrepancy to be remedied easily by incorporating various corrections. The discrepancy in case of lighter fermions are much more and can be removed only after considering radiative corrections [34-40]. We have retained a larger tolerance of 50\% in those ratios. The corresponding plots are shown in Fig.s 5 and 6. It can be seen that only a narrow range of the yukawa coupling $|f|$ at the electroweak scale leads to $b-\tau$ unification at the GUT scale whereas a wide range of $\tan \beta$ values can give rise to the same. 


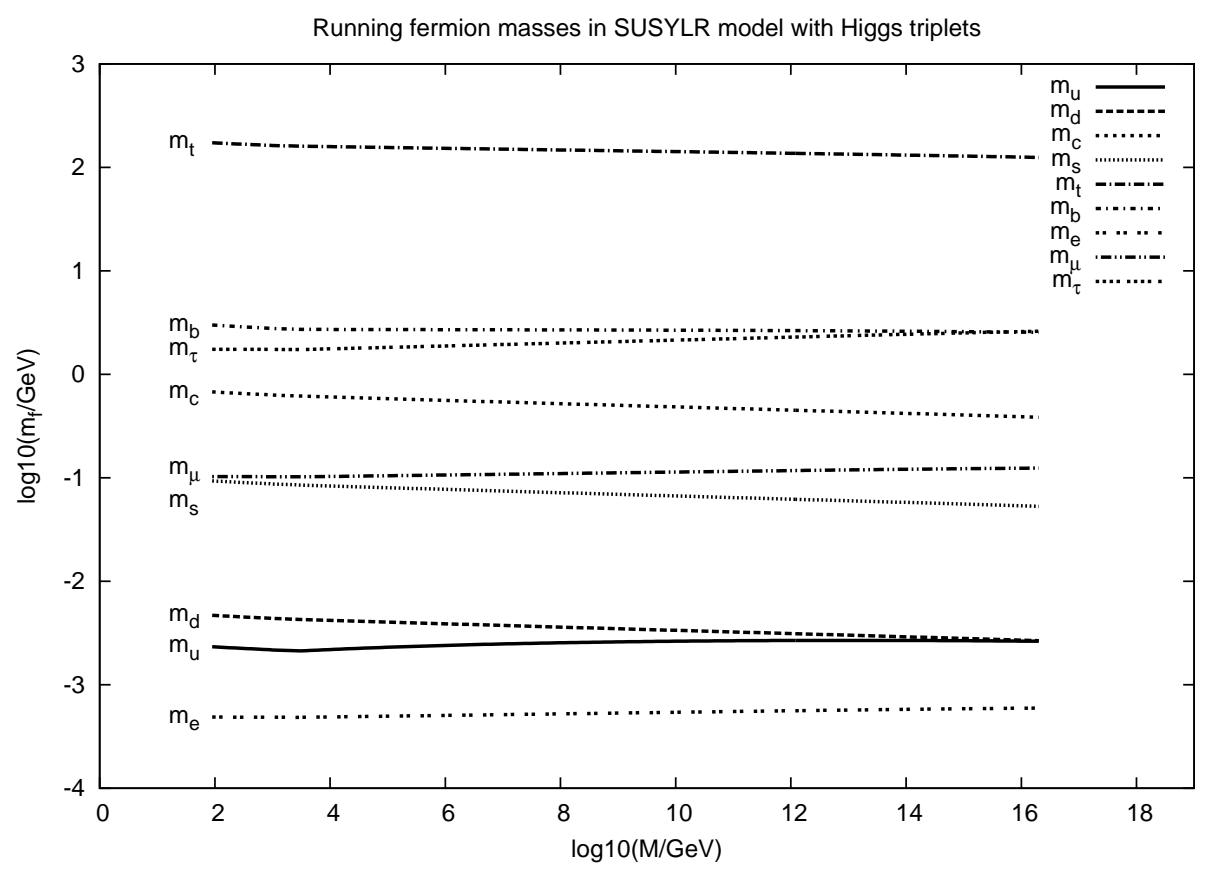

FIG. 3: Running fermion masses in the ABMRS model with extra colored superfields $\chi_{1,2}\left(3,1,1,-\frac{2}{3}\right), \bar{\chi}_{1,2}\left(\overline{3}, 1,1, \frac{2}{3}\right), M_{S U S Y}=1 \mathrm{TeV}, m_{\Omega}=M_{B-L}=3 \mathrm{TeV}, m_{\triangle}=M_{R}=M_{\mathrm{GUT}}=$ $2 \times 10^{16} \mathrm{GeV}$ and $|f|=0.55, \tan \beta=10$ at $M=M_{Z}$

\section{NEUTRINO MASS}

The type I and type II contributions to the generalised see-saw relation for the light neutrino mass matrix $m_{\nu i j}$ in the left-right models are characterised respectively as

$$
m_{\nu i j}^{I}=-\left(M_{D} M_{R}^{-1} M_{D}^{T}\right)_{i j} ; \quad m_{\nu i j}^{I I}=f_{i j} v_{L}
$$

where $M_{D}$ is the dirac mass matrix of the neutrinos $\left(M_{D}\right)_{i j}=h_{i j} v_{1}$. In the ABMRS model, as discussed below Eq.s (10)-(14) in sub-section II $v_{L}=0$ is a natural value for $\langle\triangle\rangle$, characteristic of the incorporation of supersymmetry. Thus the first term in the neutrino mass formula (27) vanishes and only the second term survives making type I seesaw natural in the ABMRS model.

In the bitriplet model the above formula (27) can be written as

$$
m_{\nu} \equiv m_{\nu}^{I I}+m_{\nu}^{I}=\frac{-f v^{2} v_{R}}{2 m_{\sigma} s}-\frac{v^{2}}{v_{R}} h f^{-1} h^{T}
$$

Attempting to keep $v_{R} \sim 1 \mathrm{TeV}$ accessible to accelerator energies, the Type II contribution to the small neutrino masses can be kept within observed limits provided $m_{\sigma} \sim s$ are at least 


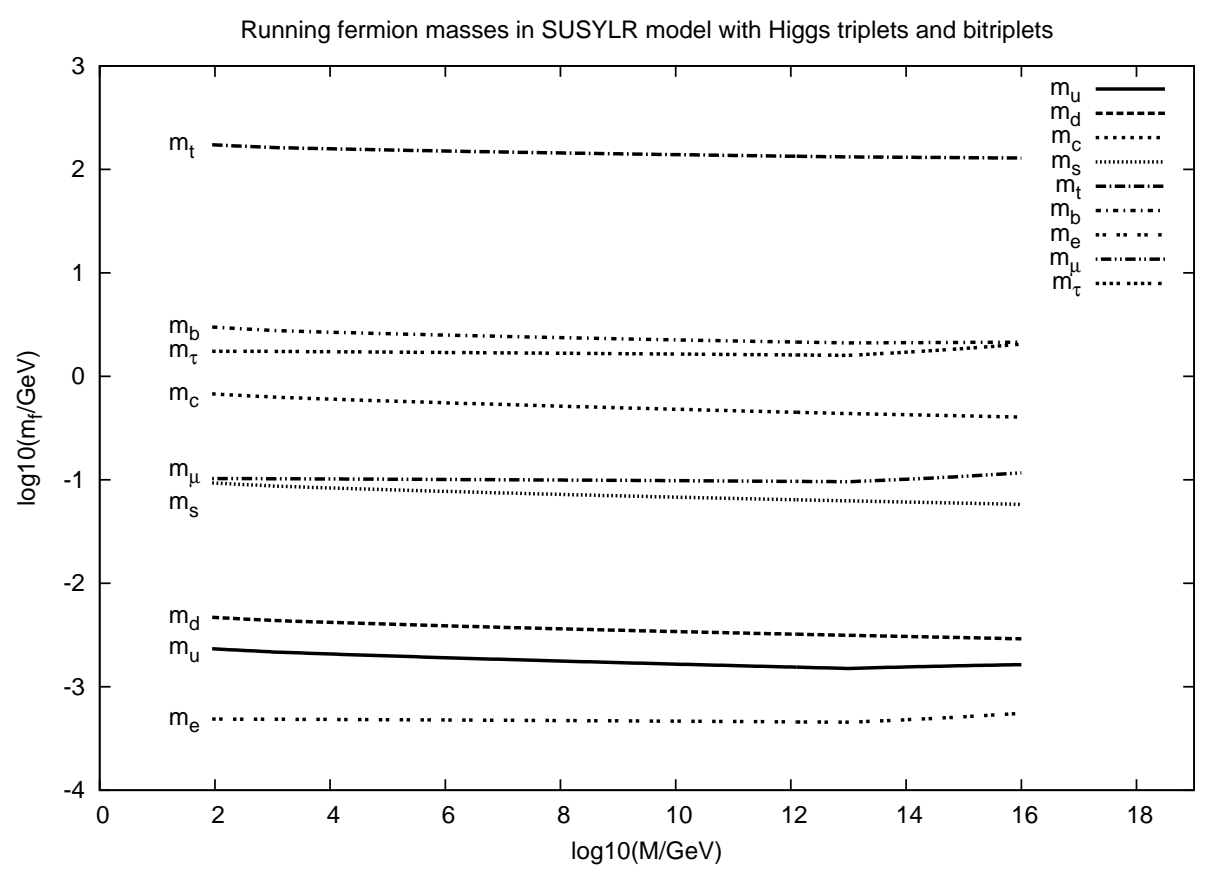

FIG. 4: Fermion masses evolution in the bitriplet model with extra colored superfields $\chi_{1,2}\left(3,1,1,-\frac{2}{3}\right), \bar{\chi}_{1,2}\left(\overline{3}, 1,1, \frac{2}{3}\right), M_{\text {susy }}=1 \mathrm{TeV}, M_{R}=5 \times 10^{12} \mathrm{GeV}, M_{\rho}=10^{16} \mathrm{GeV}$ and $|f|=0.90, \tan \beta=10$ at $M=M_{Z}$

$\gtrsim 10^{8}-10^{10} \mathrm{GeV}$. Here, as in [24], we find that the values of these mass scales should in fact be much higher, closer to GUT scale $M_{G}$ in oder to achieve gauge coupling unification. This renders the Type II contribution completely negligible even with a $v_{R}$ scale as high $\sim M_{G}$. The bitriplet model does introduce more scales than minimally required. However, the new scales introduced are stabilised by supersymmetry. This leaves open the phenomenologically interesting possibility of choosing a $\mathrm{TeV}$ scale $v_{R}$, which can potentially enter new physics signatures in collider data.

\section{RESULTS AND CONCLUSION}

Supersymmetric version of the Left-Right symmetric model including automatic Majorana mass for the neutrinos is insufficient to provide spontaneous parity breaking as a renormalizable theroy unless the Higgs structure is suitably extended. We have considered two possible extensions, ABMRS model with additional Higgs triplets and another model with an additional bitriplet as well as a parity odd singlet. In each of these we have studied 


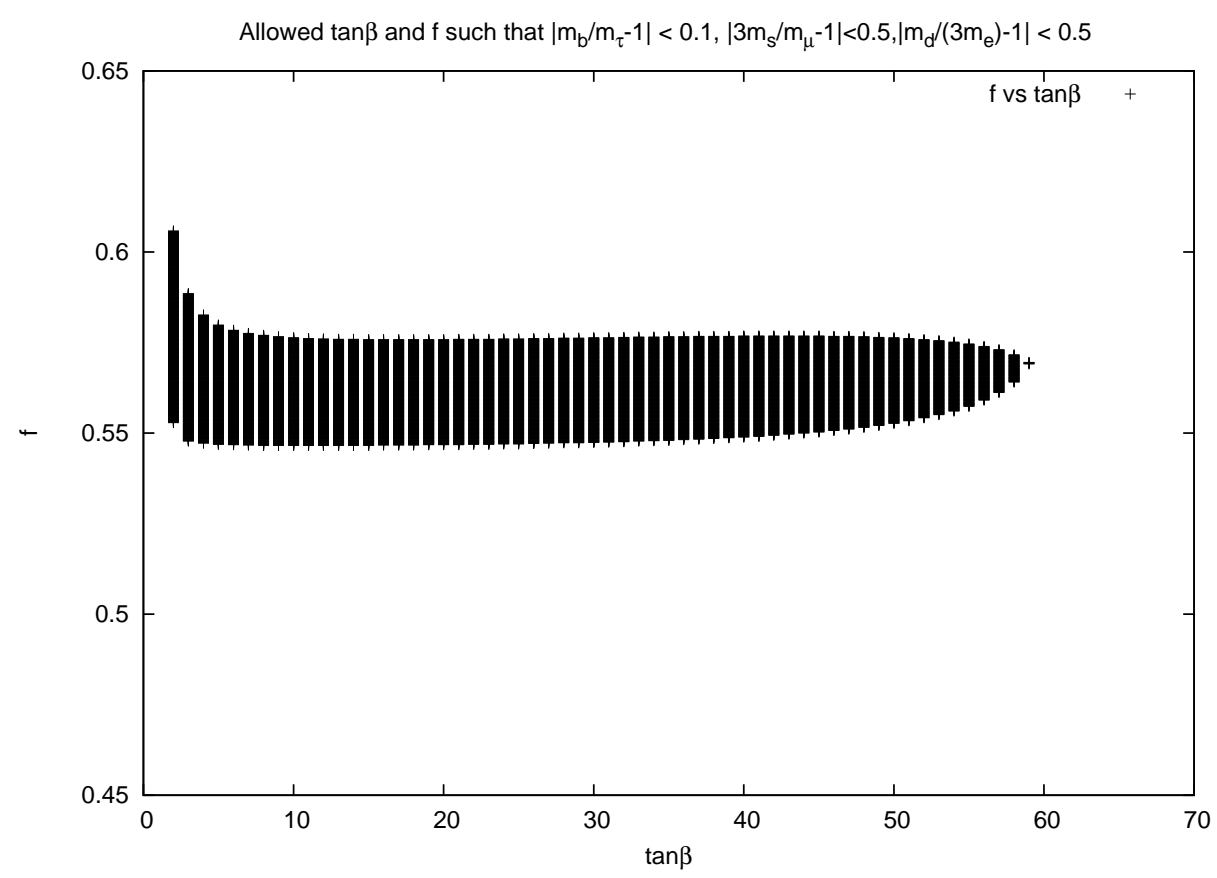

FIG. 5: Allowed parameter ranges of $f$ and $\tan \beta$ such that $\left|m_{b} / m_{\tau}\left(M_{G}\right)-1\right|<$ $0.1,\left|\frac{3 m_{s}}{m_{\mu}}\left(M_{G}\right)-1\right|<0.5,\left|\frac{m_{d}}{3 m_{e}}\left(M_{G}\right)-1\right|<0.5$ for ABMRS model with extra colored superfields $\chi_{1,2}\left(3,1,1,-\frac{2}{3}\right), \bar{\chi}_{1,2}\left(\overline{3}, 1,1, \frac{2}{3}\right), M_{S U S Y}=1 \mathrm{TeV}, m_{\Omega}=M_{B-L}=3 \mathrm{TeV}, m_{\triangle}=M_{R}=M_{\mathrm{GUT}}=$ $2 \times 10^{16} \mathrm{GeV}$

the evolution of gauge couplings and fermion masses and mixings. In the ABMRS model, a particular choice of scales $M_{B-L} \sim \mathrm{TeV}, m_{\Omega} \sim \mathrm{TeV}, m_{\triangle} \sim M_{R} \sim M_{G}$ is identified, giving rise to gauge coupling as well as $b-\tau$ unification, but which demands inclusion of two pairs of additional heavy colored superfields $\chi_{1,2}\left(3,1,1,-\frac{2}{3}\right), \bar{\chi}_{1,2}\left(\overline{3}, 1,1, \frac{2}{3}\right)$. Similar analysis in the case of the bitriplet model also requires two additional pairs of heavy colored superfields $\chi_{1,2}\left(3,1,1,-\frac{2}{3}\right), \bar{\chi}_{1,2}\left(\overline{3}, 1,1, \frac{2}{3}\right)$, which in this case decouple below the scale $M_{R}$. These extra superfields can be naturally embedded within $S O(10)$ GUT representations, $\mathbf{1 2 0}$ or $\overline{\mathbf{1 2 6}}$.

In the ABMRS case, it is possible to have a really low $M_{B-L}$ of $3 \mathrm{TeV}$, though the scale $M_{R}$ is required to be close to the Grand Unified Theory (GUT) scale. This has several interesting phenomenologically testable consequences. Firstly it makes it possible to explore some aspects of the breaking of the $B-L$ quantum number at collider energies. For example, the scale of the majorana masses of the neutrino would also be at this scale and make the physics of lepton number violation accessible to colliders. Also, this means that any baryon asymmetry of the Universe to be generated should have occurred only at a scale lower than 


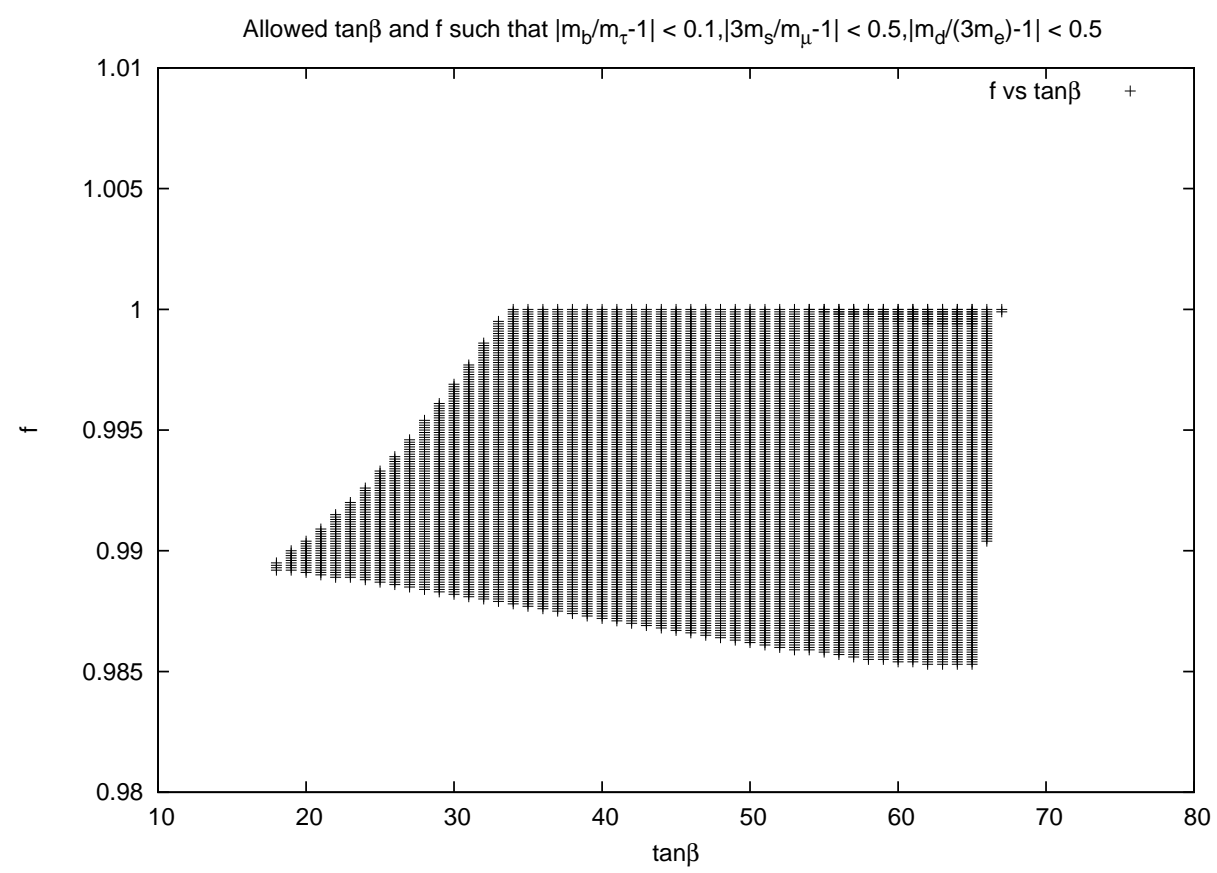

FIG. 6: Allowed parameter ranges of $f$ and $\tan \beta$ such that $\left|\frac{m_{b}}{m_{\tau}}\left(M_{G}\right)-1\right|<0.1, \mid \frac{3 m_{s}}{m_{\mu}}\left(M_{G}\right)-$ $1|<0.5,| \frac{m_{d}}{3 m_{e}}\left(M_{G}\right)-1 \mid<0.5$ for the bitriplet model with extra colored superfields $\chi_{1,2}\left(3,1,1,-\frac{2}{3}\right), \bar{\chi}_{1,2}\left(\overline{3}, 1,1, \frac{2}{3}\right), M_{S U S Y}=1 \mathrm{TeV}, M_{R}=5 \times 10^{12} \mathrm{GeV}$ and $M_{\rho}=M_{G U T}=10^{16}$ $\mathrm{GeV}$

this low scale. It has been shown that majorana masses at this scale and lighter do not conflict with baryogenesis via leptogenesis provided leptogenesis itself is non-thermal [41]. Specifically, the $C P$ violation mechanism involved in the creation of asymmetry becomes accessible to collider energies.

In the bitriplet model there appear to be several adjustable energy scales. The gauge coupling unification necessarily forces the $S U(2)_{R}$ breaking to be at a high scale $M_{R} \geq$ $5 \times 10^{12} \mathrm{GeV}$. However supersymmetry may yet protect new scales much smaller than this scale, and allow a much lower value of $v_{R}$. Existence of such new scales may provide interesting windows to new physics accessible to accelerators.

We have also identified the parameter space at the electroweak scale which gives rise to fermion mass universality at the unification scale for both the ABMRS and the bitriplet model.

Finally, we see that tiny neutrino mass arises from the type I seesaw in the ABMRS model as earlier proposed. Further, in the bitriplet model, despite the non-zero Type II 
contribution at tree level, the latter contribution is rendered utterly negligible due to the required high scale of gauge coupling unification. Thus Type I see-saw emerges as the natural explanation of the small neutrino masses in both the models.

\section{ACKNOWLEDGEMENT}

We thank Charanjit Aulakh for critical comments. This work is a part of a project supported by a grant from DST, India. UAY thanks the members of the McGill High Energy Theory group for hospitality and financial support during a sabbatical visit. DB would like to acknowledge the hospitality of Physical Research Laboratory, Ahmedabad where most of the work was done. He is also grateful to IIT Bombay for giving him a leave of absence to spend one semester at Physical Research Laboratory, Ahmedabad.

[1] J. C. Pati and A. Salam, Phys. Rev. D10, 275 (1974).

[2] R. N. Mohapatra and J. C. Pati, Phys. Rev. D11, 2558 (1975).

[3] G. Senjanovic and R. N. Mohapatra, Phys. Rev. D12, 1502 (1975).

[4] R. N. Mohapatra and R. E. Marshak, Phys. Rev. Lett. 44, 1316 (1980).

[5] N. G. Deshpande, J. F. Gunion, B. Kayser, and F. I. Olness, Phys. Rev. D44, 837 (1991).

[6] S. Fukuda et al. (Super-Kamiokande), Phys. Rev. Lett. 86, 5656 (2001), hep-ex/0103033.

[7] Q. R. Ahmad et al. (SNO), Phys. Rev. Lett. 89, 011301 (2002), nucl-ex/0204008.

[8] Q. R. Ahmad et al. (SNO), Phys. Rev. Lett. 89, 011302 (2002), nucl-ex/0204009.

[9] J. N. Bahcall and C. Pena-Garay, New J. Phys. 6, 63 (2004), hep-ph/0404061.

[10] P. Minkowski, Phys. Lett. B67, 421 (1977).

[11] M. Gell-Mann, P. Ramond, and R. Slansky (1980), print-80-0576 (CERN).

[12] T. Yanagida (1979), in Proceedings of the Workshop on the Baryon Number of the Universe and Unified Theories, Tsukuba, Japan, 13-14 Feb 1979.

[13] R. N. Mohapatra and G. Senjanovic, Phys. Rev. Lett. 44, 912 (1980).

[14] R. Kuchimanchi and R. N. Mohapatra, Phys. Rev. D48, 4352 (1993), hep-ph/9306290.

[15] C. S. Aulakh, A. Melfo, and G. Senjanovic, Phys. Rev. D57, 4174 (1998), hep-ph/9707256.

[16] K. S. Babu and R. N. Mohapatra, Phys. Lett. B668, 404 (2008), 0807.0481. 
[17] C. S. Aulakh, K. Benakli, and G. Senjanovic, Phys. Rev. Lett. 79, 2188 (1997), hep$\mathrm{ph} / 9703434$.

[18] S. Patra, A. Sarkar, U. Sarkar, and U. Yajnik, Phys. Lett. B679, 386 (2009), 0905.3220.

[19] C. S. Aulakh, A. Melfo, A. Rasin, and G. Senjanovic, Phys. Lett. B459, 557 (1999), hep$\mathrm{ph} / 9902409$.

[20] C. S. Aulakh, B. Bajc, A. Melfo, A. Rasin, and G. Senjanovic, Phys. Lett. B460, 325 (1999), hep-ph/9904352.

[21] C. S. Aulakh, B. Bajc, A. Melfo, A. Rasin, and G. Senjanovic, Nucl. Phys. B597, 89 (2001), hep-ph/0004031.

[22] J. Kopp, M. Lindner, V. Niro, and T. E. J. Underwood, Phys. Rev. D81, 025008 (2010), 0909.2653.

[23] P. S. B. Dev and R. N. Mohapatra, Phys. Rev. D81, 013001 (2010), 0910.3924.

[24] D. Borah, S. Patra, and U. Sarkar (2010), 1006.2245.

[25] M. Cvetic, Phys. Lett. B164, 55 (1985).

[26] P. Fileviez Perez and S. Spinner, Phys. Lett. B673, 251 (2009), 0811.3424.

[27] C. S. Aulakh, A. Melfo, A. Rasin, and G. Senjanovic, Phys. Rev. D58, 115007 (1998), hep$\mathrm{ph} / 9712551$.

[28] C. S. Aulakh (1997), hep-ph/9803461.

[29] D. R. T. Jones, Phys. Rev. D25, 581 (1982).

[30] N. Setzer and S. Spinner, Phys. Rev. D71, 115010 (2005), hep-ph/0503244.

[31] C. R. Das and M. K. Parida, Eur. Phys. J. C20, 121 (2001), hep-ph/0010004.

[32] C. Amsler et al. (Particle Data Group), Phys. Lett. B667, 1 (2008).

[33] H. Georgi and C. Jarlskog, Phys. Lett. B86, 297 (1979).

[34] T. Banks, Nucl. Phys. B303, 172 (1988).

[35] R. Hempfling, Phys. Rev. D49, 6168 (1994).

[36] T. Blazek, S. Raby, and S. Pokorski, Phys. Rev. D52, 4151 (1995), hep-ph/9504364.

[37] J. L. Diaz-Cruz, H. Murayama, and A. Pierce, Phys. Rev. D65, 075011 (2002), hep$\mathrm{ph} / 0012275$.

[38] S. Antusch and M. Spinrath, Phys. Rev. D78, 075020 (2008), 0804.0717.

[39] C. S. Aulakh and S. K. Garg (2008), 0807.0917.

[40] T. Enkhbat (2009), 0909.5597. 
[41] N. Sahu and U. A. Yajnik, Phys. Rev. D71, 023507 (2005), hep-ph/0410075. 